\title{
Interplay of Bipartite Entanglement between Two Geometrically Inequivalent Spin Pairs of a Spin-1/2 Heisenberg Distorted Tetrahedron
}

\author{
K. KARL'Ová* AND J. STREČKA
}

Faculty of Science, P.J. Šafárik University, Park Angelinum 9, 04001 Košice, Slovakia

\begin{abstract}
The energy spectrum, ground-state phase diagram, pair correlation functions, and concurrence are exactly calculated for a spin-1/2 quantum Heisenberg distorted tetrahedron in a magnetic field. The ground-state phase diagram in the interaction ratio versus magnetic field plane totally involves five different ground states, which differ from one another by the total spin, local pair correlation functions, and/or concurrence. In addition to the classical fully polarized ferromagnetic ground state there also appear four outstanding quantum ground states, two of which belong to a singlet sector and another two to a triplet sector. A degree of bipartite entanglement quantified through the concurrence is examined as a function of magnetic field, temperature, and the interaction ratio.
\end{abstract}

DOI: 10.12693/APhysPolA.137.595

PACS/topics: Heisenberg model, distorted tetrahedron, entanglement, concurrence

\section{Introduction}

Quantum spin systems afford important source for quantum processing of information and quantum computation [1, 2]. Molecular magnets, which involve small quantum spin clusters as a magnetic core, for instance, offer a wide possibilities for their applications in quantum computing [3]. In the present work, we will examine an interplay of bipartite entanglement between two geometrically inequivalent spin pairs in a spin- $1 / 2$ Heisenberg distorted tetrahedron, which is of particular research interest since it represents an elementary unit cell of pyrochlores [4].

\section{Model and method}

Let us consider the spin-1/2 XXZ Heisenberg distorted tetrahedron (see Fig. 1), which is defined through the following Hamiltonian:

$$
\begin{aligned}
\hat{\mathcal{H}} & =J_{1}\left[\left(\hat{\boldsymbol{S}}_{1} \cdot \hat{\boldsymbol{S}}_{2}\right)_{\Delta}+\left(\hat{\boldsymbol{S}}_{2} \cdot \hat{\boldsymbol{S}}_{3}\right)_{\Delta}+\left(\hat{\boldsymbol{S}}_{3} \cdot \hat{\boldsymbol{S}}_{4}\right)_{\Delta}+\left(\hat{\boldsymbol{S}}_{4} \cdot \hat{\boldsymbol{S}}_{1}\right)_{\Delta}\right] \\
& +J_{2}\left[\left(\hat{\boldsymbol{S}}_{1} \cdot \hat{\boldsymbol{S}}_{3}\right)_{\Delta}+\left(\hat{\boldsymbol{S}}_{2} \cdot \hat{\boldsymbol{S}}_{4}\right)_{\Delta}\right]-h \sum_{i=1}^{4} \hat{S}_{i}^{z},
\end{aligned}
$$

where $\left(\hat{\boldsymbol{S}}_{i} \cdot \hat{\boldsymbol{S}}_{j}\right)_{\Delta}=\Delta\left(\hat{S}_{i}^{x} \hat{S}_{j}^{x}+\hat{S}_{i}^{y} \hat{S}_{j}^{y}\right)+\hat{S}_{i}^{z} \hat{S}_{j}^{z}$ represents the XXZ Heisenberg interaction between the spin-1/2 particles placed at lattice sites $i$ and $j$, respectively. The exchange constants $J_{1}>0$ and $J_{2}>0$ account for antiferromagnetic interactions between two geometrically inequivalent spin pairs (see Fig. 1), $\Delta \in\langle 0 ; 1\rangle$ is an (inverse) anisotropy parameter, and the last term $h$ is the Zeeman term.

\footnotetext{
*corresponding author; e-mail: katarina.karlova@upjs.sk
}
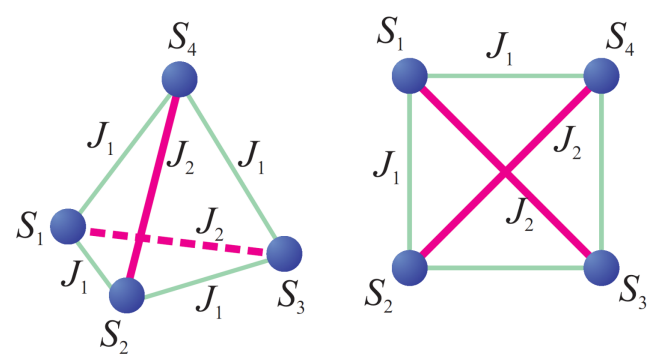

Fig. 1. A schematic illustration of the distorted tetrahedron and its equivalent planar projection. The distorted tetrahedron generally involves two geometrically inequivalent spin pairs with the site enumeration $\{1-2,1-3,3-4,4-1\}$ and $\{1-3,2-4\}$, which are coupled via the antiferromagnetic interactions $J_{1}$ and $J_{2}$, respectively.

A full energy spectrum of the spin-1/2 XXZ Heisenberg distorted tetrahedron derived after a straightforward diagonalization of the Hamiltonian (1) reads

$E_{1,2}=J_{1}+\frac{J_{2}}{2} \pm 2 h, \quad E_{3,4}=E_{5,6}=-\frac{J_{2} \Delta}{2} \pm h$,

$E_{7,8}=-J_{1} \Delta+\frac{J_{2} \Delta}{2} \pm h, \quad E_{9,10}=J_{1} \Delta+\frac{J_{2} \Delta}{2} \pm h$,

$E_{11,12}=-\frac{J_{2}}{2}, \quad E_{13}=-J_{1}+\frac{J_{2}}{2}, \quad E_{14}=-\frac{J_{2}}{2}-J_{2} \Delta$,

$E_{15,16}=-\frac{J_{1}}{2}+\frac{J_{2} \Delta}{2} \pm \frac{1}{2} \sqrt{\left(J_{1}-J_{2}+J_{2} \Delta\right)^{2}+8\left(J_{1} \Delta\right)^{2}}$.

The partition function of a spin- $1 / 2$ XXZ Heisenberg distorted tetrahedron is then given by $\mathcal{Z}=\sum_{i=1}^{16} \exp \left(-\beta E_{i}\right)$ with $\beta=1 /\left(k_{\mathrm{B}} T\right)$. All other important quantities can be derived from the partition function. A bipartite entanglement between two geometrically inequivalent spin pairs can be quantified via the quantum concurrence $[1,2,5]$ : 


$$
\begin{aligned}
& C_{12}= \max \left\{0 ; 4\left|\left\langle\hat{S}_{i}^{x} \hat{S}_{i+1}^{x}\right\rangle\right|\right. \\
&\left.-2 \sqrt{\left(\frac{1}{4}+\left\langle\hat{S}_{i}^{z} \hat{S}_{i+1}^{z}\right\rangle\right)^{2}-\left(\frac{1}{2}\left\langle\hat{S}_{i}^{z}+\hat{S}_{i+1}^{z}\right\rangle\right)^{2}}\right\}, \\
&(i=1-4, \bmod 4), \\
& C_{13}=\max \left\{0 ; 4\left|\left\langle\hat{S}_{i}^{x} \hat{S}_{i+2}^{x}\right\rangle\right|\right. \\
&\left.-2 \sqrt{\left(\frac{1}{4}+\left\langle\hat{S}_{i}^{z} S_{i+2}^{z}\right\rangle\right)^{2}-\left(\frac{1}{2}\left\langle\hat{S}_{i}^{z}+\hat{S}_{i+2}^{z}\right\rangle\right)^{2}}\right\}, \\
&(i=1,2),
\end{aligned}
$$

which is expressed through two spatial components of the pair correlation and local magnetization

$$
\begin{aligned}
& \left\langle\hat{S}_{i}^{x} \hat{S}_{i+1}^{x}\right\rangle=-\frac{1}{8} \frac{\partial \ln \mathcal{Z}}{\partial\left(\beta J_{1} \Delta\right)}, \quad\left\langle\hat{S}_{i}^{z} \hat{S}_{i+1}^{z}\right\rangle=-\frac{1}{4} \frac{\partial \ln \mathcal{Z}}{\partial\left(\beta J_{1}\right)}, \\
& \left\langle\hat{S}_{i}^{x} \hat{S}_{i+2}^{x}\right\rangle=-\frac{1}{4} \frac{\partial \ln \mathcal{Z}}{\partial\left(\beta J_{2} \Delta\right)}, \quad\left\langle\hat{S}_{i}^{z} \hat{S}_{i+2}^{z}\right\rangle=-\frac{1}{2} \frac{\partial \ln \mathcal{Z}}{\partial\left(\beta J_{2}\right)}, \\
& \frac{1}{2}\left\langle\hat{S}_{i}^{z}+\hat{S}_{i+1}^{z}\right\rangle=\frac{1}{2}\left\langle\hat{S}_{i}^{z}+\hat{S}_{i+2}^{z}\right\rangle=-\frac{1}{4} \frac{\partial \ln \mathcal{Z}}{\partial(\beta h)}
\end{aligned}
$$

\section{Results and discussion}

Let us proceed to a discussion of the most interesting results for a spin- $1 / 2$ Heisenberg distorted tetrahedron by considering the particular case with the isotropic coupling $(\Delta=1)$. The ground-state phase diagram is depicted in Fig. 2 in the $J_{2} / J_{1}-h / J_{1}$ plane. As one can see, the ground-state phase diagram involves five different ground states discernible according to the quantum concurrence between two geometrically inequivalent spin pairs. In addition, all ground states differ from one another by quantum spin numbers related to the total spin $S_{T}=S_{1}+S_{2}+S_{3}+S_{4}$ and two composite spin pairs $S_{13}=S_{1}+S_{3}$ and $S_{24}=S_{2}+S_{4}$. The relevant ground-state eigenvectors $\left|S_{T}, S_{13}, S_{24}\right\rangle$ take the following explicit form:

$$
\begin{aligned}
&|2,1,1\rangle=\left|\uparrow_{1} \uparrow_{2} \uparrow_{3} \uparrow_{4}\right\rangle, \\
&|0,0,0\rangle=\frac{1}{2}\left(\left|\uparrow_{1} \uparrow_{2} \downarrow_{3} \downarrow_{4}\right\rangle-\left|\uparrow_{1} \downarrow_{2} \downarrow_{3} \uparrow_{4}\right\rangle-\left|\downarrow_{1} \uparrow_{2} \uparrow_{3} \downarrow_{4}\right\rangle\right. \\
&\left.+\left|\downarrow_{1} \downarrow_{2} \uparrow_{3} \uparrow_{4}\right\rangle\right), \\
&|1,1,0\rangle=\frac{1}{\sqrt{2}}\left(\left|\uparrow_{1} \uparrow_{2} \uparrow_{3} \downarrow_{4}\right\rangle-\left|\uparrow_{1} \downarrow_{2} \uparrow_{3} \uparrow_{4}\right\rangle\right), \\
&|1,0,1\rangle=\frac{1}{\sqrt{2}}\left(\left|\uparrow_{1} \uparrow_{2} \downarrow_{3} \uparrow_{4}\right\rangle-\left|\downarrow_{1} \uparrow_{2} \uparrow_{3} \uparrow_{4}\right\rangle\right), \\
&|1,1,1\rangle=\frac{1}{2}\left(\left|\uparrow_{1} \uparrow_{2} \uparrow_{3} \downarrow_{4}\right\rangle-\left|\uparrow_{1} \uparrow_{2} \downarrow_{3} \uparrow_{4}\right\rangle+\left|\uparrow_{1} \downarrow_{2} \uparrow_{3} \uparrow_{4}\right\rangle\right. \\
&\left.-\left|\downarrow_{1} \uparrow_{2} \uparrow_{3} \uparrow_{4}\right\rangle\right),
\end{aligned}
$$

$$
\begin{aligned}
& |0,1,1\rangle=\frac{1}{\sqrt{3}}\left(\left|\uparrow_{1} \downarrow_{2} \uparrow_{3} \downarrow_{4}\right\rangle+\left|\downarrow_{1} \uparrow_{2} \downarrow_{3} \uparrow_{4}\right\rangle\right) \\
& -\frac{1}{\sqrt{12}}\left(\left|\uparrow_{1} \uparrow_{2} \downarrow_{3} \downarrow_{4}\right\rangle+\left|\uparrow_{1} \downarrow_{2} \downarrow_{3} \uparrow_{4}\right\rangle+\left|\downarrow_{1} \uparrow_{2} \uparrow_{3} \downarrow_{4}\right\rangle\right. \\
& \left.\quad+\left|\downarrow_{1} \downarrow_{2} \uparrow_{3} \uparrow_{4}\right\rangle\right) .
\end{aligned}
$$

To bring a deeper insight into a bipartite entanglement between the spin pairs coupled via the interaction $J_{1}$ we will comprehensively examine the concurrence $C_{12}$ as a function of the magnetic field and temperature in three different cuts of the parameter space. It can be seen from Fig. 3 that the low-temperature asymptotes $\left(k_{\mathrm{B}} T / J_{1}=0.001\right)$ of the concurrence $C_{12}$ are consistent with the ground-state phase diagram (Fig. 2). In fact, the concurrence $C_{12}$ is in general kept constant within the individual ground states, while it often shows abrupt changes in vicinity of field-driven phase transitions. It is interesting that the concurrence exhibits for the interaction ratio $J_{2} / J_{1}=0.5$ an abrupt decrease (increase) just below (above) the field-induced phase transition between the ground states $|0,1,1\rangle$ and $|1,1,1\rangle$ with the same value of the concurrence $C_{12}$ at low enough temperature $k_{\mathrm{B}} T / J_{1}=0.05$, whereas this nonmonotonous trend is retained up to relatively high temperatures $k_{\mathrm{B}} T / J_{1} \cong 0.2$ (see Fig. 3a). The concurrence $C_{12}$ is displayed in Fig. 3b for the special case $J_{2} / J_{1}=1.0$ without any distortion. As one can see, the rising temperature plays role of the inertia, i.e., the higher the temperature is, the higher is the delay against the magnetic-field change. Finally, the concurrence $C_{12}$ for the interaction ratio $J_{2} / J_{1}=1.5$ is zero independently of temperature due to a singlet pairing of spins coupled with the interaction $J_{1}$ within the ground state $|0,0,0\rangle$.

The field dependences of the concurrence $C_{13}$ of other two geometrically inequivalent spin pairs is depicted in Fig. 4 for the same set of parameters as used before for the concurrence $C_{12}$. For the interaction ratio $J_{2} / J_{1}=0.5$ one finds that the concurrence $C_{13}$ is

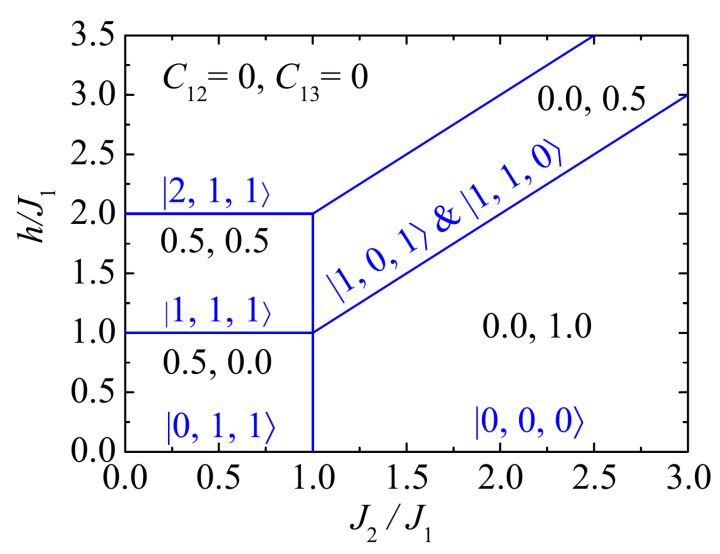

Fig. 2. The ground-state phase diagram of a spin- $1 / 2$ Heisenberg distorted tetrahedron, which consists of five different eigenvectors $\left|S_{T}, S_{13}, S_{24}\right\rangle$. Two numbers determine the quantum concurrence $C_{12}$ and $C_{13}$. 

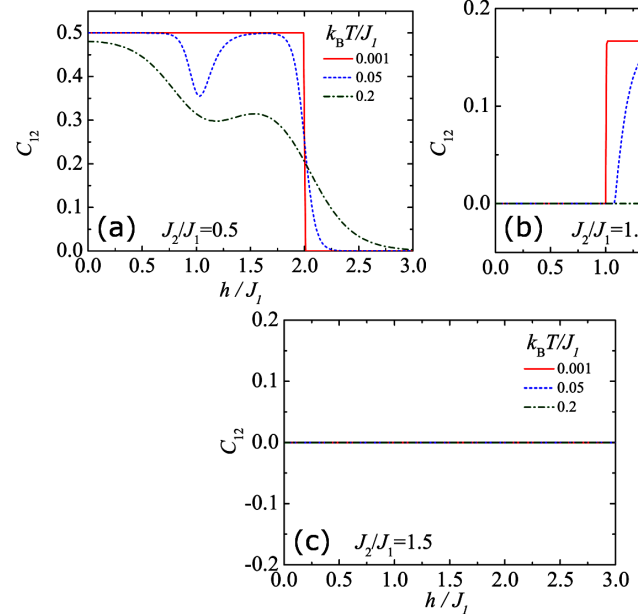

Fig. 3. The field dependences of the concurrence $C_{12}$ for three different temperatures and the interaction ratio: (a) $J_{2} / J_{1}=0.5$, (b) $J_{2} / J_{1}=1.0$, (c) $J_{2} / J_{1}=1.5$.
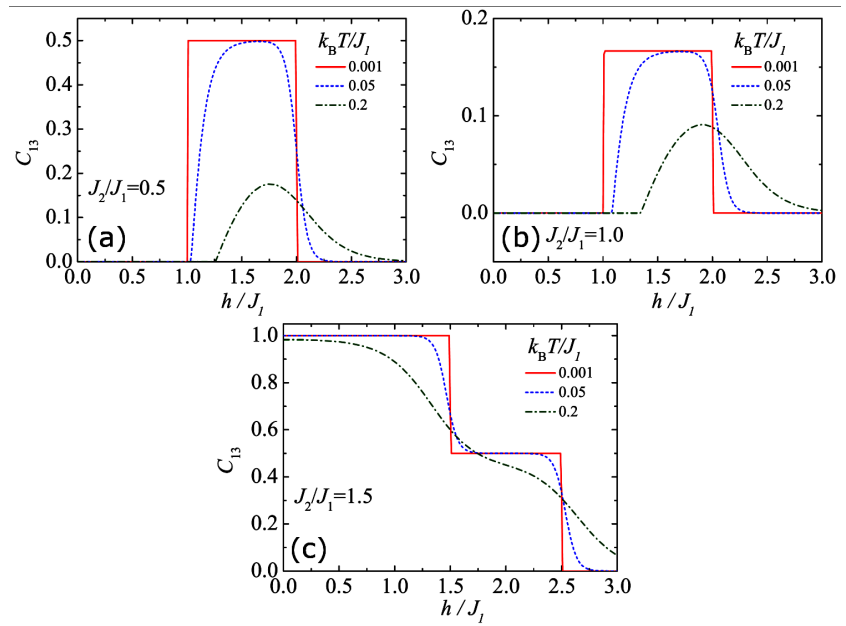

Fig. 4. The field dependences of the concurrence $C_{13}$ for three different temperatures and the interaction ratio: (a) $J_{2} / J_{1}=0.5$, (b) $J_{2} / J_{1}=1.0$, (c) $J_{2} / J_{1}=1.5$.

nonzero at very low temperatures $\left(k_{\mathrm{B}} T / J_{1}=0.001\right)$ just within the ground state $|1,1,1\rangle$ emergent at moderate magnetic fields $h / J_{1} \in(1,2)$, while at higher temperatures it may become nonzero also above the saturation field due to thermal excitations from the fully polarized state $|2,1,1\rangle$ (Fig. 4a). If the interaction ratio is set to $J_{2} / J_{1}=1.0$, the spin- $1 / 2$ Heisenberg tetrahedron lacks any spatial anisotropy and hence, the concurrence $C_{13}$ becomes identical with the concurrence $C_{12}$ in agreement with general expectations (cf. Fig. 4b with Fig. 3b). Lastly, the concurrence $C_{13}$ is plotted in Fig. 4c for the interaction ratio $J_{2} / J_{1}=1.5$. Obviously, the concurrence achieves its maximal value at very low temperature within the ground state $|0,0,0\rangle$ due to a singlet pairing between spins coupled with the interaction $J_{2}$. Contrary to this, the concurrence reaches half of its maximal value $\left(C_{13}=0.5\right)$ within twofold degenerate ground state $|1,1,0\rangle /|1,0,1\rangle$, because one spin pair undergoes a singlet pairing and the other one is fully polarized.

\section{Conclusions}

In the present work we have studied an interplay of bipartite entanglement between two geometrically inequivalent spin pairs of a spin- $1 / 2$ Heisenberg distorted tetrahedron. We have shown that the spin- $1 / 2$ Heisenberg distorted tetrahedron exhibits five different ground states, one of which is twofold degenerate. The microscopic nature of all ground states was clarified. Moreover, we have found that all ground states are discernible according to the concurrence between two geometrically inequivalent spin pairs with exception of a very special case of the spatially isotropic spin-1/2 Heisenberg tetrahedron without any distortion.

\section{Acknowledgments}

This work was supported by grant Nos. VEGA 1/0531/19, APVV-16-0186, vvgs-2017-652, and European Regional Development Fund under the project ITMS: $313011 T 544$.

\section{References}

[1] L. Amico, R. Fazio, A. Osterloh, V. Vedral, Rev. Mod. Phys. 80, 517 (2008).

[2] R. Horodecki, P. Horodecki, M. Horodecki, K. Horodecki, Rev. Mod. Phys. 81, 865 (2009).

[3] J. Schnack, Dalton Trans. 39, 4677 (2010).

[4] J.S. Gardner, M.J.P. Gingras, J.E. Greedan, Rev. Mod. Phys. 39, 53 (2010).

[5] W.K. Wooters, Phys. Rev. Lett. 80, 2245 (1998). 